\title{
Visualizing Differences between Two Large Graphs
}

\author{
Markus Geyer, Michael Kaufmann, and Robert Krug \\ Wilhelm-Schickard-Institut für Informatik, Universität Tübingen, Germany \\ \{geyer, mk, krug\}@informatik. uni-tuebingen.de
}

\section{Introduction}

When working with graphs one often faces the problem of comparing two or more graphs. For example in biology, when two protein-protein interaction networks from closely related species have to be compared, the graphs can easily contain several hundred nodes and thousands of edges but very few differences. Our goal was to develop a software-tool that creates an overview of large graphs and maintains the structure but reduces the number of nodes and edges and enables the user to easily find and investigate the areas of interest. This problem has been considered in the past by various groups ([1], [2]) and different heuristics have been proposed. Here, we follow the concept proposed in [3]. For this work, we assume that the input-graphs are relatively large with small local differences and node correspondences are known.

\section{Our Method}

\section{Condensation of Graphs}

We denote the condensation of a graph as the extraction of its structure and the reduction to its important parts. Our first approach identifies important nodes and removes the remaining nodes from the graph. To assess the importance of a node we used different centrality measures from [4]. Neighboring nodes are merged into group nodes as long as their combined centrality value doesn't exceed a pre-defined threshold.

For the second approach a given number of nodes with the highest centrality value is chosen and all other nodes are merged with the closest chosen node. In both cases new edges have to be created to maintain connectivity.

In order to enable the user to investigate interesting areas of the graph, a detailed view of a part of the graph can be created by clicking on a node in the condensed graph. An extra window is then opened which shows the area in the immediate proximity of the chosen node in uncondensed form. By clicking on one of the dashed lines, which represent edges to parts of the graph not yet displayed, the currently shown graph-part can be extended into that direction. Also, by clicking on a normal edge, the subgraph beginning at the target-node of that edge can be removed from the detailed view.

\section{Comparison of Graphs}

For the construction of the overview graph the common elements are identified and compacted using one of the previously described methods. Then, for each difference a node is added. For the resulting graph a layout is computed with a force-directed 
method. Clicking on one of the difference nodes opens another window that shows the difference and the area around it in detail. It is also possible to extend and contract the part of the graph that is currently shown. For the detailed view, the positions of elements from the overview graph stays the same while new elements are placed with a force-directed method. Once a node has been set to a position, it will always stay there to ease orientation around the graph for the user.

\section{Implementation and Results}

For our implementation we used Java and the $y$ Files graph library ([5]). Figure 1 depicts the overview graph before and after the condensation as well as a detailed view of a difference node.

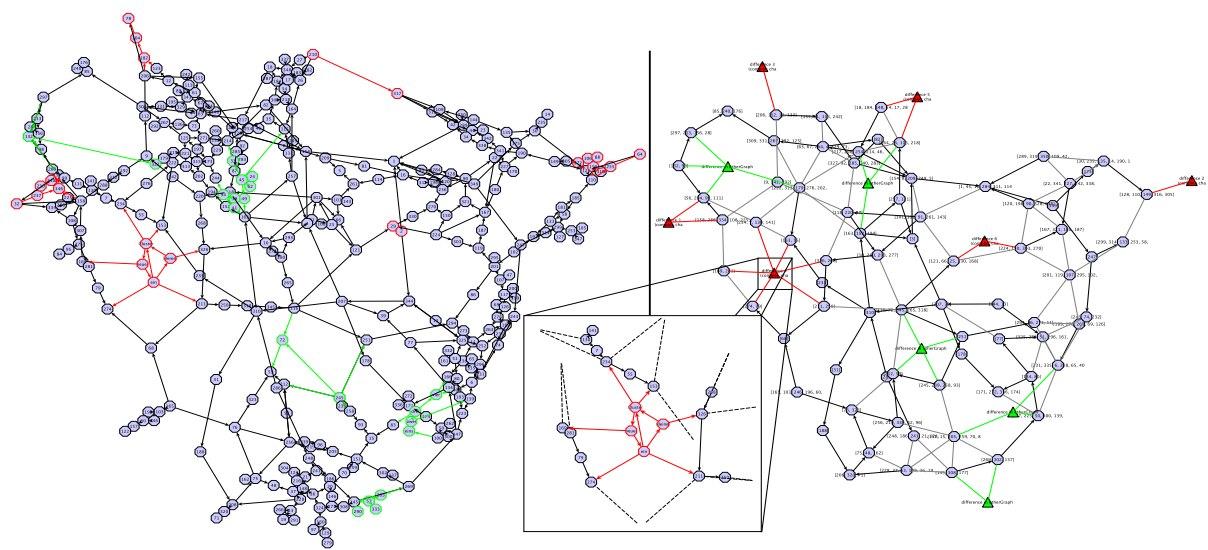

Fig. 1. The overview graph (left) contains 350 nodes while the condensed version (right) contains 75 blue group nodes and 11 red or green difference nodes. In the detailed view in the middle colored elements indicate differences and dashed lines expansion edges.

\section{References}

[1] Chimani, M., Jünger, M., Schulz, M.: Crossing minimization meets simultaneous drawing. In: PacificVis, pp. 33-40. IEEE, Los Alamitos (2008)

[2] Tan, K., Shlomi, T., Feizi, H., Ideker, T., Sharan, R.: Transcriptional regulation of protein complexes within and across species. Proceedings of the National Academy of Sciences 104(4), 1283-1288 (2007)

[3] Albrecht, M., Estrella-Balderrama, A., Geyer, M., Gutwenger, C., Klein, K., Kohlbacher, O., Schulz, M.: Visually comparing a set of graphs. In: Borgatti, S.P., Kobourov, S., Kohlbacher, O., Mutzel, P. (eds.) Graph Drawing with Applications to Bioinformatics and Social Sciences, Dagstuhl Seminar Proceedings, Dagstuhl, Germany, vol. 8191 (2008)

[4] Freeman, L.C.: Centrality in social networks: Conceptual clarification. Social Networks 1(3), 215-239 (1979)

[5] yWorks GMbH: yFiles graph library, http://www.yworks.com 